GEORG HENRIK VON WRIGHT 


\section{APUNTES SOBRE LA VIDA Y LA OBRA DE GEORG HENRIK VON WRIGHT (1916-2003)*}

Daniel González Lagier

Universidad de Alicante

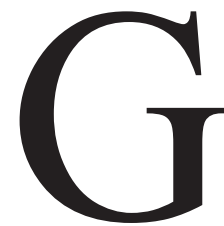

eorg Henrik von Wright nació en Helsinki el 14 de Junio de 1916 y falleció en esa misma ciudad el 16 de Junio de 2003, a la edad de 87 años. Fue un filósofo y un lógico de enorme influencia, que contribuyó al esclarecimiento conceptual de un buen número de problemas filosóficos, y al mismo tiempo un intelectual y ensayista profundo, que en su empeño de no ceder al dogmatismo sometió a revisión una y otra vez los presupuestos culturales de nuestro modo de ver el mundo.

Aunque vivió gran parte de su vida en Finlandia, en su infancia pasó largas temporadas en Brooklyn y en Merano (Austria), donde aprendió alemán. Este idioma fue muy importante para su desarrollo intelectual y le permitió un conocimiento temprano de la poesía y la prosa de Goethe, Schiller, Schopenhauer, Nietzsche, Kafka, Musil, etc. En 1934 entró en la Universidad de Helsinki y se graduó tres años después. Allí conoció a Eino Kaila, uno de los líderes intelectuales de Finlandia, quien, junto con Wittgenstein y Moore, ejerció sobre von Wright una gran influencia filosófica. Kaila había sido miembro del Círculo de Viena y le introdujo en el pensamiento de Russell, Wittgenstein, Carnap, Popper, Hempel, Keynes, Dubislav, Reichenbach, von Mises, Neurath, Waismann, Schlick, etc.; le enseñó el uso de la lógica formal, le proporcionó un método y lo inició en la "lógica filosófica". Von Wright ha dicho que debe la forma de su filosofía a Eino Kaila y el contenido a Ludwig Wittgenstein.

* Este texto es un resumen y actualización de Daniel González Lagier, Acción y norma en G.H. von Wright, Centro de Estudios Constitucionales, 1995, págs 23-43. Ha sido publicado también en Theoria, vol. 19, núm. 49, Enero 2004, págs. 107-114. Las citas entrecomilladas son de G. H. von Wright, Intellectual Autobiography, en The Philosophy of Georg Henrik von Wright, The Library of Living Philosophers, vol. XIX, 1989. 
Tras graduarse viajó a Cambridge para realizar una tesis doctoral sobre la justificación de la inducción. Von Wright considera a Cambridge como "su segundo hogar intelecual"; allí conoció personalmente a Moore y a Wittgenstein, pasó diversas etapas de su vida y ocupó una cátedra (la del propio Wittgenstein) desde 1948 hasta 1951. Este primer encuentro con Wittgenstein fue decisivo para la orientación filosófica de von Wright y su abandono del empirismo lógico (que había sido la filosofía de sus años de estudiante). Con el inicio de la guerra mundial, von Wright regresó a Finlandia.

En 1943 fue Docent en la Universidad de Helsinki, donde años más tarde llegó a tener una cátedra propia. Durante los años de posguerra su actividad docente fue muy intensa y muchos de sus libros tienen su origen en algún curso. Uno de sus estudiantes más destacados de esa etapa fue Jaakko Hintikka. En 1947 von Wright volvió a Cambridge, donde en esta ocasión conoció a Elizabeth Anscombe, Peter Geach y Norman Malcolm y se encontró nuevamente con Wittgenstein, que estaba impartiendo su último curso antes de retirarse. Este último le habló sobre sus dudas acerca de abandonar la cátedra y le preguntó si estaría de acuerdo en ser su sucesor. En 1948 Wittgenstein dejó efectivamente la cátedra y von Wright ocupó su lugar durante tres años y medio. En esos años surgió su interés por la lógica modal y la lógica deóntica y profundizó en la historia y la filosofía de la ciencia; mantuvo discusiones regulares con Peter Geach, Alan Anderson, Knut Erik Tranoy y también con Moore, con quien discutía sobre lógica filosófica y de quien le impresionó su rigor y honestidad. Wittgenstein continuaba yendo a Cambridge de tanto en tanto, hasta su muerte, alojándose en casa de von Wright. La relación entre ambos fue muy estrecha.

Poco después de la muerte de Wittgenstein (1951), von Wright volvió a Finlandia y permaneció en la Universidad de Helsinki hasta 1961, momento en que se incorporó a la Academy of Finland. Esto le supuso una liberación de las obligaciones docentes, lo que le permitió dedicarse a la organización de seminarios, coloquios, etc. y a numerosas visitas a distintas universidades estadounidenses y europeas: ha sido profesor visitante en la Universidad de Cornell -considerada por él su "tercer hogar intelectual”, después de Helsinki y Cambridge - de 1965 a 1977 y en la de Leipzig durante el curso 19941995. Ha recibido numerosos premios (como el Alexander von Humboldt o el gran premio de la Academia de Suecia) y fue nombrado doctor honoris causa por distintas universidades. Su actividad intelectual continuó siendo intensa hasta poco antes de su fallecimiento.

\section{II}

La obra de von Wright sorprende por sus dimensiones y su diversidad. Por ello sólo podemos limitarnos a dar cuenta de algunas de sus aporta- 
ciones más relevantes. Por otro lado, resulta difícil dividir su trayectoria intelectual en etapas, puesto que von Wright siempre se ha caracterizado por someter sus ideas a una continua reelaboración, lo que hace que sus líneas de investigación permanezcan siempre abiertas.

Sus primeras investigaciones versaron sobre inducción y probabilidad (su primera publicación sobre este tema fue el artículo On Probability ${ }^{1}$ ). Este fue también el tema de su tesis doctoral. En 1941 publicó esta tesis con el título de The Logical Problem of Induction ${ }^{2}$. El interés de von Wright en la inducción había sido -a pesar del título- más bien de carácter epistemológico, por lo que algunas cuestiones formales habían quedado, en su opinión, descuidadas. Su insatisfacción por esos descuidos le impulsó a continuar en esta dirección. Para ello trató de aclarar las nociones de condición necesaria y condición suficiente y su aplicación a la inducción (en lugar de operar con las nociones de causa y efecto, como era habitual en esta materia). Todo ello toma forma -tras otras publicaciones anteriores- en el libro $A$ Treatise on Induction and Probability.

Tras su segunda visita a Cambridge, su trabajo creativo se extiende fuera de la órbita de la inducción y la probabilidad. Su nuevo objetivo era la noción de verdad lógica, que estudia en On the Idea of Logical Truth $I^{4}$ y On the Idea of Logical Truth $I I^{5}$. En ellos desarrolla las formas normales distributivas como un método de decisión de la verdad de las fórmulas lógicas y las teorías de la cuantificación simple y doble. Von Wright quedó insatisfecho de estos resultados, pero pronto le resultaron útiles: "Un día, temprano, en 1949, mientras paseaba a orillas del Cam, observé la siguiente analogía: de la misma manera que los cuantificadores 'alguno', 'ninguno' y 'todos' están relacionados unos con otros, así también están relacionadas las modalidades 'posible', 'imposible' y 'necesario"'. Los dos grupos de conceptos también compartían sus características distributivas respecto a la conjunción y la disyunción, por lo que parecía posible desarrollar una lógica modal en analogía con la lógica de los cuantificadores. De esta manera, von Wright construyó su propio sistema de lógica modal. Pero esta analogía podía extenderse a otros conceptos, por ejemplo las nociones epistémicas

\footnotetext{
${ }^{1}$ Von Wright, On Probability, en Mind, 1940.

${ }^{2}$ Von Wright, The Logical Problem of Induction, Acta Philosophica Fennica, Helsinki, 1941.

${ }^{3}$ Von Wright, A Trataise of Induction and Probability, International Library of Psychology, Philosophy and Scientific Method, Londres, Routledge and Kegan Paul, 1951.

${ }^{4}$ Von Wright, On the Idea of Logical Truth I, en Societas Scientiarum Fennica. Commentationes Physico-Mathematicae, vol. 14, núm. 4, Helsinki, 1948.

${ }^{5}$ Von Wright, On the Idea of Logical Truth II, en Societas Scientiarum Fennica. Commentationes Physico-Mathematicae, vol. 15, núm. 10, Helsinki, 1950.
} 
("verificado", “indeterminado" y "falsado") y las deónticas ("obligatorio", "permitido" y "prohibido"). Todo parecía apuntar a la posibilidad de construir una "teoría general de las modalidades" (las modalidades aléticas serían un tipo más de modalidad lógica); además, la lógica modal podría construirse, no como una alternativa a la lógica proposicional (como habían hecho Lukasiewicz y C.I. Lewis), sino como una "superestructura" basada en la lógica proposicional (como la lógica de la cuantificación), lo que permitía generalizar a las distintas "ramas" de esa lógica modal la aplicación de tablas de verdad y de formas normales distributivas. El fruto de estas ideas es el libro An Essay in Modal Logic ${ }^{6}$. En 1951 von Wright también publicó en Mind un artículo -basado en la analogía entre los conceptos aléticos y los deónticos- cuya difusión fue inmediata y que se considera el punto de partida de la lógica de las normas: Deontic Logic ${ }^{7}$.

De vuelta en Helsinki, von Wright abre una nueva dirección en sus investigaciones. Se propone ahora discutir algunos tópicos especiales de la lógica filosófica. Este es el origen de una serie de trabajos sobre los condicionales (On Conditionals ${ }^{8}$ ), la implicación (The Concept of Entailment ${ }^{9}$ ), la negación (On the Logic of Negation ${ }^{10}$ ), etc. De nuevo, iniciar una línea de investigación no supone para nuestro autor abandonar las anteriores y continúa también su trabajo en lógica modal, tratando de desarrollar modalidades relativas ( $A$ New System of Modal Logic ${ }^{11}$ ), lo que parecía de especial importancia para la lógica deóntica ( A New System of Deontic Logic ${ }^{12}$ ), y de extenderla a los conceptos valorativos (On the Logic of Some Axiological and Epistemological Concepts ${ }^{13}$ ). Este último intento resultó fallido, en opinión de von Wright: la relación entre los opuestos axiológicos (como el bien y el mal) es de un tipo distinto de la relación entre opuestos modales

\footnotetext{
${ }^{6}$ Von Wright, An Essay in Modal Logic, en Studies in the logic and the Foundations of Mathematics, núm. 4, Amsterdam, 1951.

${ }^{7}$ Von Wright, Deontic Logic, en Mind, núm. 60, 1951.

${ }^{8}$ Von Wright, On Conditionals, en von Wright, Logical Studies, International Library of Psychology, Philosophy and Scientific Method, Routledge and Kegan Paul, Londres, 1957.

${ }^{9}$ Von Wright, The concept of Entailment, en von Wright, Logical Studies, International Library of Psychology, Philosophy and Scientific Method, Routledge and Kegan Paul, Londres, 1957.

${ }^{10}$ Von Wright, On the Logic of Negation, Societas Scientiarum Fennica. Commentationes Physico-Mathematicae, vol. 22, núm. 4, Helsingfors, 1959.

${ }^{11}$ Von Wright, A New System of Modal Logic, Actas del XI International Congress of Philosophy (Bruselas), Logic, Philosophical Analysis, Philosophy of Mathematics, Amsterdam-Lovaina, 1953.

${ }^{12}$ Von Wright, A New System of Deontic Logic, en von Wright, Logical Studies, International Library of Psychology, Philosophy and Scientific Method, Routledge and Kegan Paul, Londres, 1957.

${ }^{13}$ Von Wright, On the Logic of Some Axiological and Epistemological Concepts, en Ajatus, núm. 17, 1952.
} 
(como necesario e imposible u obligatorio y prohibido). Nuestro autor siguió ocupándose del tema y publicó años después The Logic of Preference: An Essay ${ }^{14}$ y The Logic of Preference Reconsidered ${ }^{15}$.

$\mathrm{Su}$ interés por la lógica deóntica y sus clases en la cátedra de filosofía moral (que simultaneó con la suya propia durante un tiempo) le condujeron a la ética y a la teoría general de las normas y valores. De la invitación a dar las Gifford Lectures proceden dos de los libros más conocidos de von Wright: Norm and Action ${ }^{16}$ y The Varieties of Goodness ${ }^{17}$. Ambos libros han hecho de von Wright un autor enormemente influyente en la filosofía del Derecho y la filosofía moral.

Norm and action es el resultado de una permanente reflexión sobre los problemas planteados por la lógica deóntica desde su nacimiento; la perspectiva adoptada por von Wright es, más que formal, de carácter ontológico. Quizá lo más importante del libro no es el sistema de lógica deóntica desarrollado en él (que pretende ser, ante los problemas de la aplicabilidad de los valores verdadero/falso a las normas, una lógica de proposiciones normativas, esto es, de proposiciones sobre la existencia de las normas, y no directamente una lógica de normas), sino el estudio conceptual de la noción de norma, de su estructura, de las clases o tipos o sentidos en los que se puede hablar de normas, de las condiciones de existencia de las mismas, de la noción de validez, etc. Von Wright ha continuado revisando las ideas expuestas en este libro (no sólo las de índole formal, sino también las relativas a la ontología de las normas). Por ejemplo en Deontic Logic and the Theory of Conditions ${ }^{18}$ y On the Logic and Ontology of Norms ${ }^{19}$.

The varieties of Goodness (en su opinión, su libro más personal y mejor argumentado) no es propiamente un libro de ética prescriptiva, sino que su pretensión es "tejer la red conceptual que constituye nuestro 'punto de vista moral"'. En este libro von Wright propone, frente a la tesis de la autonomía conceptual de la moral, que los conceptos de bondad o corrección moral deben ser estudiados en relación con los conceptos que se refieren "al hombre como un todo", como, por ejemplo, los de felicidad, salud y bienestar. El de bienestar es el concepto más importante del grupo: el "bienestar" podría ser llamado también "bien del hombre". Desde esta perspectiva, "la moralidad

\footnotetext{
${ }^{14}$ Von Wright, The Logic of Preference: An Essay, Edinburgh University Press, 1963.

${ }^{15}$ Von Wright, The Logic of Preference Reconsidered, en Theory and Decision, núm. 3,

${ }^{16}$ Von Wright, Norms and Action, Routledge and Kegan Paul, Londres, 1963.

${ }^{17}$ Von Wright, The Varieties of Goodness, Routledge and kegan Paul, Londres, 1963.

${ }^{18}$ Von Wright, Deontic Logic and the Theory of Conditions, en Critica, núm. 2, 1968.

${ }^{19}$ Von Wright, On the Logic and Ontology of Norms, en von Wright, Philosophical Logic,
} 1972. Ed. J.W. Davis, D. Reidel, Dordrecht-Holland, 1969. 
de la conducta es una función de cómo la conducta de un individuo afecta al bienestar de sus compañeros humanos", "la moralidad es necesariamente una idea social".

Ambos libros incluyen el germen de posteriores desarrollos. Por un lado, Norm and action dio lugar a una serie de nuevas investigaciones en lógica deóntica y abrió la investigación en la lógica de la acción (una de las conclusiones de este libro es que una lógica deóntica debe construirse sobre la lógica de la acción). En Un ensayo de lógica deóntica y la teoría general de la acción ${ }^{20}$ se exponen nuevas ideas sobre ambas lógicas y se inicia un desplazamiento de la atención de von Wright desde la lógica de la acción y del cambio a la noción de tiempo, que dio lugar a trabajos como And $N e x t^{21} \mathrm{y}$ And Then ${ }^{22}$. Por otro lado, con The Varieties of Goodness comienza una serie de trabajos sobre la inferencia práctica. Aunque la teoría del razonamiento práctico es también "lógica práctica", es independiente de la lógica deóntica y de la lógica de la acción. La inferencia práctica se ocupa de la relación entre el pensamiento y la acción (von Wright, en la línea de Aristóteles, afirma que la conclusión de esta inferencia es una acción o la disposición a realizar una acción); es lógica -dice von Wright- "en algún sentido diferente y quizá más libre".

El silogismo práctico es la pieza clave de un grupo de estudios de von Wright. Por un lado, von Wright ha tratado de analizar mejor la naturaleza de este silogismo en algunos trabajos (Practical Inference ${ }^{23}$; On so-called Practical Inference ${ }^{24}$ ); por otro lado, el silogismo práctico permite lidiar con uno de los problemas fundamentales de la filosofía, con implicaciones para la cuestión del método propio de las ciencias humanas: la naturaleza de la relación entre la intención y la acción. Si esta relación es causal, la explicación de las acciones ha de seguir el patrón de las explicaciones causales que se realizan de los sucesos de la naturaleza; si no es causal, el modelo de explicación de las acciones ha de ser distinto. Von Wright argumenta que la relación entre acción e intención es conceptual y que al igual que el modelo de explicación "nomológico-deductivo" es el más característico de las ciencias de la naturaleza, el silogismo práctico es el modelo de explicación característico de las ciencias humanas. Estas ideas son expuestas en Expla-

${ }^{20}$ Von Wright, An Essay in Deontic Logic and the General Theory of Action, North-Holland Publishing Co., Amsterdam, 1968. Acta Philosophica Fennica, fasc. 21.

${ }^{21}$ Von Wright, And Next, en Studia Logico-Mathematica et Philosophica in Honorem Rolf Nevanlinna, Acta Philosophica Fennica, Helsinki, 1965.

${ }^{22}$ Von Wright, And Then, en Societas Scientiarum Fennica. Commentationes Physico-Mathematicae, vol. 32, núm. 7, Helsingfors, 1966.

${ }^{23}$ Von Wright, Practical Inference, en Philosophical Review, núm. 72, 1963.

${ }^{24}$ Von Wright, On so-called Practical Inference, en Acta Sociologica, núm. 15, 1972. 
nation and Understanding ${ }^{25}$. En este libro -quizá el más conocido de von Wright-, además, podemos encontrar un estudio de la noción de causa del que caben destacar dos cosas: en primer lugar, su utilización de la teoría de las condiciones para profundizar en el comportamiento de la relación causal; en segundo lugar, la dependencia conceptual que esta noción adquiere en el tratamiento de von Wright frente a la idea de acción humana. Estas dos ideas (causas como condiciones y la relación conceptual entre causa y acción) se desarrollan con mayor profundidad en Causality and Determinism ${ }^{26}$.

La discusión acerca de la acción, la causalidad y la explicación lleva de una manera casi ineludible a discutir cuestiones como el determinismo y el libre albedrío. Para von Wright el determinismo relativo a la conducta del hombre -el determinismo en las ciencias humanas- no es el mismo tipo de determinismo que encontramos en las ciencias de la naturaleza. Debemos distinguir entre estímulos internos y externos de la conducta y debemos analizar el tipo de relación que existe entre tales estímulos y la acción. Estas cuestiones son estudiadas -entre otros trabajos- en Determinism and the Study of Man $^{27}$, Freedom and Determination ${ }^{28}$ y Explanation and Understanding of Action ${ }^{29}$.

Hacia los años 80 von Wright retoma los temas de lógica filosófica -impulsado por algunas cuestiones que habían quedado pendientes en su primera incursión en ella- con una serie de investigaciones sobre lo que podría titularse genéricamente -es el título de una conferencia presentada en Oxford en 1978- Tiempo, verdad y necesidad. Son de esta época, por ejemplo, Determinism and Knowledge of the Future ${ }^{30} \mathrm{y}$ Determinism and Future Truth ${ }^{31}$. Al mismo tiempo, von Wright no ha abandonado su trabajo en lógica deóntica y teoría de la norma; muestra de ello son On the Logic

${ }^{25}$ Von Wright, Explanation and Understanding, Cornell Contemporary Philosophy Series, International Library of Philosophy and Scientific Method, Ithaca, N.Y. Cornell University Press, 1971.

${ }^{26}$ Von Wright, Causality and Determinism, Columbia University Press, Nueva York y Londres, 1973.

${ }^{27}$ Von Wright, Determinism and the Study of Man, en Essays on Explanation and Understanding, Ed. Juha Manninem y Raimo Tuomela, Synthese Library, 72, D. Reidel, Dordrecht-Holland, 1976.

${ }^{28}$ Von Wright, Freedom and Determination, en Acta Philosophica Fennica, vol. 31, núm. 1, North-Holland Publishing Co., Amsterdam, 1980.

${ }^{29}$ Von Wright, Explanation and Understanding of Action, en Revue Internationale de Philosophie, núm. 35, 1981.

${ }^{30}$ Von Wright, , Determinism and Knowledge of the Future, en Tulevaisuuden Tutkimuksen Seuran Julkaisu, 4, Turku, 1982.

${ }^{31}$ Von Wright, Determinism and Future Truth, en Truth, Knowldedge and Modality, Philosophical Papers, vol. 3, Basil Blackwell, Oxford, 1984. 
of Norms and Actions ${ }^{32}$, Norms, Truth and Logic ${ }^{33}$, Is and Ought ${ }^{34}$, Is There a Logic of Norms $?^{35}$... En Norms, Truth and Logic von Wright expuso lo que -durante un tiempo- consideró su "ajuste de cuentas" final con la lógica de las normas. En Is There a Logic of Norms? encontramos reformuladas algunas de aquellas opiniones (aunque no sustancialmente). Algunas de las últimas posiciones de von Wright sobre lógica filosófica (incluyendo la posibilidad de la lógica deóntica) pueden encontrarse en su libro Six Essays in Philosophical Logic ${ }^{36}$.

Simultáneamente, los estudios sobre la causalidad, la explicación intencional y, sobre todo, el determinismo acaban conduciendo a von Wright a la filosofía de la mente, especialmente al problema de la relación entre la mente y el cuerpo. Fruto de este interés es In The Shadow of Descartes. Essays in the Philosophy of Mind, publicado en $1998^{37}$. En él destaca el ensayo On Human Freedom ${ }^{38}$, que constituye un intento de aunar su teoría de la acción, su concepción de la explicación intencional, el problema de la relación entre la mente y el cuerpo y la libertad humana.

Además de este tipo de filosofía, von Wright se ha ocupado también de cuestiones como el progreso, la tecnología, el estado del mundo, la racionalidad, etc. La mayoría de sus trabajos sobre estas cuestiones han sido publicadas en sueco y finlandés, pero algunas de ellas fueron recogidas en The Tree of Knowledge and Other Essays ${ }^{39}$. Este es un libro especialmente recomendado para conocer los dos tipos de actividad filosófica y de pensamiento que marcan la trayectoria intelectual de von Wright. El mismo lo considera, en cierto sentido, una "autobiografía intelectual". El libro tiene dos partes: La primera de ellas nos permite conocer al "filósofo analítico von Wright" a través de tres vías distintas: (1) a través de sus comentarios acerca del desarrollo de la filosofía analítica y la lógica a lo largo de este siglo; (2) a través de sus observaciones sobre los dos filósofos que más han

\footnotetext{
${ }^{32}$ Von Wright, On the Logic of Norms and Actions, en Resto Hilpinen (Ed.), New Studies in Deontic Logic, D. Reidel, Dordrecht-Holland, 1981.

${ }^{33}$ Von Wright, Norms, Truth and Logic, en A.A. Martino (ed.), Deontic Logic, Computational Linguistics and Legal Information Systems, North-Holland, Amsterdam, 1982.

${ }^{34}$ Von Wright, Is and Ought, en E. Bulygin (ed.), Man, Law and Modern Forms of Life, D. Reidel, Dordrecht-Holland, 1985.

${ }^{35}$ Von Wright, Is There a Logic of Norms?, en Ratio Iuris, vol. 4, núm. 3, Diciembre 1991.

${ }^{36}$ Von Wright, Six Essays in Philosophical Logic, Acta Philosophica Fennica, vol. 60, 1006.

${ }^{37}$ Von Wright, In the Shadow of Descartes. Essays in the Philosophy of Mind, Kluwer Academic Publishers, Dordrecht/Boston/London, 1998.

${ }^{38}$ VON Wright, On Human Freedom, publicado originalmente en The Tanner Lectures on Human Values, vol. VI, University of Utah Press, Salt Lake City, 1985.

${ }^{39}$ Von Wright, The Tree of Knowledge and Other Essays, E.J. Brill, 1993.
} 
influido en su trayectoria: Eino Kaila y Wittgenstein; y (3) a través de su propia visión de sus aportaciones al análisis conceptual y a la lógica. La segunda de ellas nos presenta la vertiente humanista de su filosofía, mucho menos conocida, en la que von Wright se plantea -partiendo de una revisión de nuestros presupuestos culturales- cuestiones como las siguientes: ¿Puede realmente afirmarse que la dirección en la que la ciencia empuja al hombre es la mejor para éste? ¿Qué legitima al progreso científico por encima de otros tipos de "progreso"? ¿Qué es en realidad el progreso? Sus respuestas tienen a menudo un tinte pesimista.

Para finalizar con la actividad filosófica de von Wright, no debe omitirse la labor que ha realizado en la edición de las obras de Wittgenstein. Cuando falleció, Wittgenstein sólo había publicado el Tractatus y un artículo. La tarea de encontrar sus notas y ordenarlas debió ser considerable. En colaboración con otros autores (normalmente con G.E.M. Anscombe y Rush Rhees), von Wright ha editado importantes trabajos de Wittgenstein y ha elaborado un calendario (The Wittgenstein Papers ${ }^{40}$ ) que se ha convertido en un punto de origen de cualquier discusión sobre los escritos de este autor.

En toda la obra de von Wright se aprecia una constante actitud de rehuir el dogmatismo, de dar por sentado algún presupuesto. Sus trabajos siempre tienen la pretensión de ser meros "ensayos": llegar a conclusiones definitivas sólo puede ser el resultado de un lento proceso de análisis y corrección. Por eso puede decirse de la obra de von Wright lo que él mismo dijo de la de Wittgenstein: “A veces he pensado que lo que convierte en clásica la obra de un hombre es precisamente esta multiplicidad que a la vez incita y repele a nuestra ansia de claro entendimiento".

${ }^{40}$ Von Wright, The Wittgenstein Papers, en Philosophical Review, núm. 78, 1969. 